\title{
A New Adaptive Method for Target Tracking in Wireless Sensor Networks
}

\author{
Elham Ahmadi \\ Department of Computer \\ Engineering, Islamic Azad \\ University,South Tehran \\ Branch, \\ Tehran, Iran
}

\author{
Masoud Sabaei \\ Department of Computer \\ Engineering and Information \\ Technology, Amirkabir \\ University, \\ Tehran, Iran
}

\author{
Mohamad Hosain Ahmadi \\ Department of Electronic \\ Engineering, \\ Smart Company \\ Tehran, Iran
}

\begin{abstract}
These days mobile target tracking is considered as one of the important applications of wireless sensor networks. In this regard, the clustering structure is one of the most applicable network structures. In this paper, we suggested a new method for target tracking that makes adaptation with target mobility model. This method utilizes two tools to create adaptability which are changing the size and shape of clusters according to target mobility model. Also by combining static and dynamic clustering a semi dynamic clustering structure has been developed to implement these tools. Simulation results show that our suggested method decreases both energy consumption by decreasing clusters size when the target moves uniformly, and tracking error by changing the size and the shape of clusters according to target mobility when the target moves unpredictably.
\end{abstract}

\section{General Terms}

Semi-Dynamic clustering, target tracking, tracking error.

\section{Keywords}

Wireless sensor networks, target tracking, tracking error, target mobility model.

\section{INTRODUCTION}

Sensor network (WSN) is composed of a large number of sensor nodes and deployed either inside the phenomenon or very close to it. Wireless sensor networks are expected serve as a key infrastructure for a broad range of applications including precision agriculture, surveillance highway systems, emergent disaster response and recovery. One of the important application issues for sensor networks is utilized to track mobile object [1]. Researches about target tracking with continuous monitoring mechanism can be divided into three categories: structure-less scheme, tree-based scheme, and cluster-based scheme. In structure-less scheme, when a node detects an object within its range, it broadcasts a 'TargetDetected' message. This message contains the location of the sensor node and the distance to the target. All nodes that hear this message store its data in their local memory. When a node that has detected the target hears two other 'TargetDetected' messages from two other nodes, it performs triangulation on the three coordinates to calculate the location of the target [2]. Tree-based scheme uses a hierarchy to connect the sensors. In this scheme, when a target shows up for the first time, an initial convey tree is constructed and the root

collects data from nodes surrounding the target, and processes the data. When the target moves, the tree's membership is changed and its structure is reconfigured if necessary [3].
To facilitate collaborative data processing in target tracking centric sensor networks, the cluster architecture is usually used in which sensors are organized into clusters, and each cluster consists of a cluster head $(\mathrm{CH})$ and several neighboring sensors (members). In the static clustering architecture, clusters are formed statically at the time of network deployment. The attributes of each cluster, such as the size of a cluster, the area it covers, and the members it possesses, are static. In spite of its simplicity, this structure suffers from several drawbacks; for example, it can't adapt itself with the average error of tracking because of the fixed number of nodes in each cluster. Also if a $\mathrm{CH}$ dies of power depletion, all the sensors in the cluster render useless [4].

In dynamic clustering structure, when target moves, the nodes that recognize the target gradually form dynamic clusters. This method is more accurate than static clustering structure but has higher calculation and communication overhead [4, 5]. In summary, the clustering architecture is more applicable for tracking applications and it has less overhead rather than other structures [4]

Regarding these cases, we decided to suggest a new clustering structure that, in contrast with static clustering, can change clusters size according to tracking error value and have less calculation and communication overhead rather than dynamic clustering. In addition, few researches of target tracking are concerned with the target mobility model type. Therefore, we decided to suggest a new tracking protocol that is adaptable with the target mobility model.

The remaining of this paper is organized as follows. In section 2, we first review some related works. In section 3, our proposed target tracking method is introduced and described. In section 4 , we compare energy consumption in our structure with other clustering structures (static and dynamic clustering). In section 5 , we analyze and evaluate this approach throughout simulation. Finally, we conclude the paper in section 6 with a summary.

\section{RELATED WORKS}

Paper [6] has proposed a density based clustering for node management that is a decentralized algorithm having the topology control information in each sensor node. The authors in [7] have proposed an object tracking strategy named multi level object tracking (MLOT) which is based on multi-level architecture for efficient object tracking and real-time recovery of missing objects by mining the movement $\log$ in sensor networks. In [8] a distributed protocol for target tracking based on static clustering has been developed that predicts the target's next location using a linear predictor. Also an efficient dynamic clustering algorithm for object tracking in [9] has been introduced that proposes a new cluster head election algorithm 
and helps to minimize the overlap area between clusters. In [10], a new classification algorithm has been proposed that provides easy classification based on the velocity estimate. It also provides a way to reduce the uncertainty in the movement of the target and thus the target's position estimation error is reduced. In [11], an efficient dynamic clustering mechanism has been introduced which reduces energy consumption in two ways: first, minimizing the number of nodes involved in tracking by constructing the cluster dynamically along the target's traveling route. Second, minimizing the communication cost between sensor nodes when forming a cluster.

\section{PROPOSED METHOD}

Most tracking protocols suggested up to now [1, 2, 3, 4] have not noticed the type of target mobility model sufficiently. In other words, is the efficiency of a tracking protocol the same when tracking a tank or a human? In summary, the question is whether the efficiency of a suggested tracking protocol is dependent on the target's mobility parameters. In order to answer this question, we suggested a new tracking method that adapts with the target's mobility parameters by using a flexible clustering structure. Our suggested method makes adaptation by utilizing two tools: changing the size and shape of the clusters regarding the target mobility model. In the following, we are going to study these two tools in detail.

\subsection{The effect of target mobility model on the size of clusters}

In this section, the effect of clusters size on the accuracy of target tracking protocol in different mobility models is going to be investigated. For example, consider two scenarios. In the first scenario, the target moves in a Random Waypoint Model and in the second, it moves in a Random Directed Model. Also, let's consider the network is based on a static clustering structure. The results of simulations (Figure 1) show that while the target moves uniformly, increasing or decreasing the number of nodes in clusters has a little effect on tracking error. So it is favorable to decrease the number of nodes in each cluster in order to reduce the energy consumption. On the other hand, incrementing the number of nodes in each cluster is very effective in reducing tracking error when the target moves in Random Waypoint Model. In other words it is preferable to increase the clusters size when the target move in Random Mobility Model and decrease it for situations where the target moves in Random Directed Model. By analyzing the simple example described above, we decided to develop a new method that can adapt itself with various mobility models, and be able to provide acceptable performance. In the following, we are going to describe our proposed protocol in detail.

\subsection{The effect of target mobility model on the shape of clusters}

In this section, we study the relation between the target mobility and the cluster shape. Consider two scenarios. In the first scenario a person walks, and in the second, a target like a motorcycle moves with a high velocity. When a person is walking, at every moment, he can turn right or left easily and does not have inertia in its movement. If this person at time ${ }^{t}$ be in $x(t)$, we can join this fact and the knowledge of target velocity to define $P(x(t+1) \mid x(t))$ as a disk centered at $x(t)$

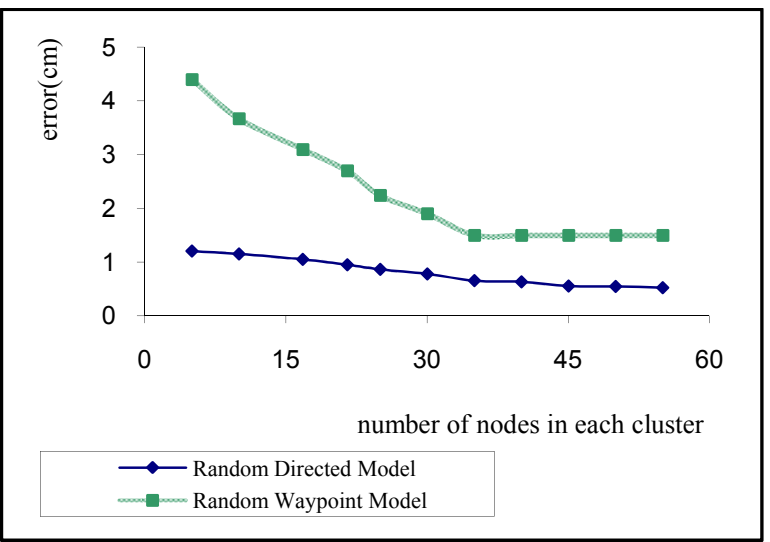

Fig. 1. The effect of the number of nodes in each cluster on error in various mobility models

with radius $v(t)[10]$.

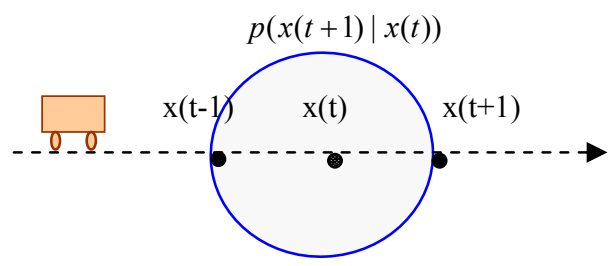

Fig. 2. $p(x(t+1) \mid x(t))$ of a target which has a low velocity

On the other side, there are fast targets. They have quick movements and it produces that they can't change their movement direction at each instant. For example, when a motorcycle moves with high velocity, it needs more time than a person to turn right or left without problems. These targets have inertia in their movement. If you suppose that this target moves with a velocity in $\operatorname{range}\left(v_{\min }, v_{\max }\right)$, then we can define $p(x(t+1) \mid x(t))$ as an ellipse which the focal points are $\left(x_{\min }(t+1)\right)$ and $\left(x_{\max }(t+1)\right)$.

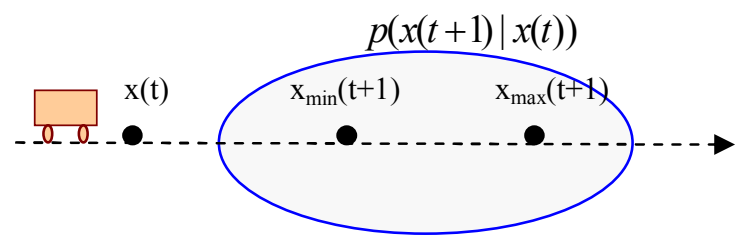

Fig. 3. $p(x(t+1) \mid x(t))$ of a target which has a high velocity

Regarding Figures 2 and 3, we can consider wake up areas as an ellipse, the long diameter depends on velocity changes, and its short diameter depends on direction changes. Generally, to track a target that moves uniformly, wake up areas are in elliptical form with a short diameter, but on the other hand to track a 
target moving unforeseeably, the wakening up areas are in circular form. In the next sections, we study this tool in detail.

\subsection{Network structure in proposed method}

In the previous sections, we concluded that in target tracking, the type of target mobility model impacts on the size and shape of clusters so we try to adapt the size and shape of clusters according to target mobility model. In other words, we intend to implement the adaptation tools by using a clustering structure. Therefore, we seek a clustering structure that can form dynamic clusters with respect to target mobility parameters and previous tracking error values during the target's movement.

Our proposed structure in this paper is a Semi-Dynamic Clustering structure (SDC). At first, the network's backbone is defined as static clustering. Later during the tracking some of these backbone clusters are merged regarding the number of nodes needed for tracking the target. One of the important features of this structure is that we have two cluster head levels. The first-level includes the cluster head nodes of backbone clusters, named $\mathrm{CH} 1$, and the second-level cluster head node (referred to as $\mathrm{CH} 2$ ) which is usually located at the next predicted location of target.

After receiving information from member nodes in backbone clusters, the $\mathrm{CH} 1$ nodes send their integrated information to the $\mathrm{CH} 2$ node, which integrates the received information from all $\mathrm{CH} 1$ nodes and transmits the integrated information to the base station. A scheme of SDC structure has been shown in Figure 4.

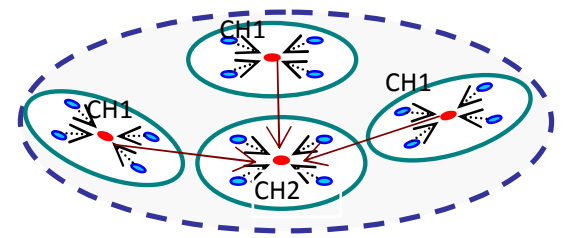

Fig. 4. A scheme of a new cluster formed

\subsection{Approximation of wakening up area in the next sampling time}

According to Figure 5 suppose the location of the mobile object at the instance of $t+1$ is approximately predicted by estimating the target's velocity during the time $[t-1, t]$.

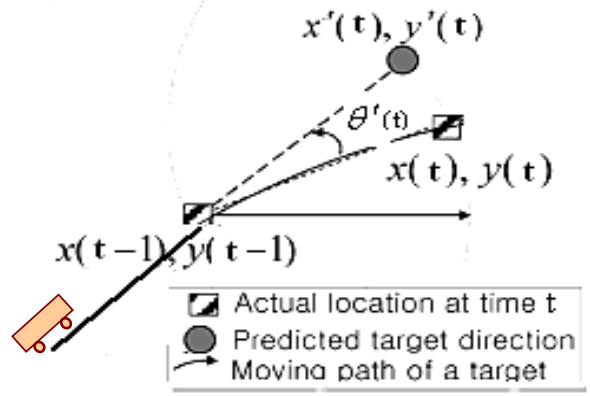

Fig. 5. A scenario of target tracking in wireless sensor networks
Let us suppose the target's location at the time instants $T_{t-1}$ and $T_{t}$ is $(x(t-1), y(t-1))$ and $(x(t), y(t))$, then the target's velocity according to [12] can be estimated as:

$$
v_{\text {pre }}(t+1)=\frac{\sqrt{(x(t)-x(t-1))^{2}+(y(t)-y(t-1))^{2}}}{(t)-(t-1)}
$$

Suppose we express the prediction error by the angle between the actual location and the previously predicted one, denoted by $\theta^{\prime}(t)$ as shown in Figure 5. Then we have

$$
\begin{aligned}
& \cos \theta^{\prime}(t)=\frac{x^{\prime}(t)-x(t-1)}{\sqrt{\left[x^{\prime}(t)-x(t-1)\right]^{2}+\left[y^{\prime}(t)-y(t-1)\right]^{2}}} \\
& -\frac{x(t)-x(t-1)}{\sqrt{[x(t)-x(t-1)]^{2}+[y(t)-y(t-1)]^{2}}}
\end{aligned}
$$

Then we can predict the new moving direction of the mobile target by:

$$
\begin{aligned}
\cos \theta(t+1)= & \frac{x^{\prime}(t)-x(t-1)}{\sqrt{\left[x^{\prime}(t)-x(t-1)\right]^{2}+\left[y^{\prime}(t)-y(t-1)\right]^{2}}} \\
& -\cos \theta^{\prime}(t)
\end{aligned}
$$

In addition to the predicted velocity which is $v_{\text {pre }}(t+1)$, we also consider the maximum and minimum velocity $\left(v_{\max }(t+1)\right.$, $\left.v_{\text {min }}(t+1)\right)$ based on the error estimation, $e_{v}$ as:

$$
\begin{gathered}
v_{\text {min }}(t+1)=v_{\text {pre }}(t+1)-e_{v} \\
v_{\text {max }}(t+1)=v_{\text {pre }}(t+1)+e_{v}
\end{gathered}
$$

Then the target's location based on $v_{\text {pre }}(t), v_{\min }(t)$ and $v_{\max }(t)$ is calculated as:

$$
\begin{aligned}
& L_{\text {pre }}(t+1)=\left[\begin{array}{l}
x_{\text {pre }}(t+1) \\
y_{\text {pre }}(t+1)
\end{array}\right]=\left[\begin{array}{l}
x(t)+v_{\text {pre }}(t+1) \operatorname{Cos} \theta(t+1) \\
y(t)+v_{\text {pre }}(t+1) \operatorname{Sin} \theta(t+1)
\end{array}\right] \\
& L_{\text {min }}(t+1)=\left[\begin{array}{l}
x_{\text {min }}(t+1) \\
y_{\text {min }}(t+1)
\end{array}\right]=\left[\begin{array}{l}
x(t)+v_{\text {min }}(t+1) \operatorname{Cos} \theta(t+1) \\
y(t)+v_{\text {min }}(t+1) \operatorname{Sin} \theta(t+1)
\end{array}\right] \\
& L_{\text {max }}(t+1)=\left[\begin{array}{l}
x_{\text {max }}(t+1) \\
y_{\text {max }}(t+1)
\end{array}\right]=\left[\begin{array}{l}
x(t)+v_{\text {max }}(t+1) \operatorname{Cos} \theta(t+1) \\
y(t)+v_{\text {max }}(t+1) \operatorname{Sin} \theta(t+1)
\end{array}\right]
\end{aligned}
$$

Considering these relations, the long diameter of waking up's cluster in the next sampling time, denoted by $J_{x}(t+1)$ is defined as:

$$
J_{x}(t+1)=\left|L_{\max }(t+1)-L_{\min }(t+1)\right|
$$

Also, the short diameter of the wake up cluster in the next sampling time, denoted by $J_{y}(t+1)$, will be

$$
J_{y}(t+1)=\alpha J_{y}(t)+(1-\alpha)\left(c v_{p r e}(t)\left|\tan \theta^{\prime}(t)\right|\right)+b
$$

where 
- $J_{y}(t+1)$ is the short diameter size of waking up cluster in sampling time $t+1$.

- $J_{y}(t)$ is the short diameter size of waked up cluster in sampling time $t$.

- $\alpha$ is the effective rate of waked up cluster at sampling time $t$ on the size of waking up cluster at sampling time $t+1$ ( $\alpha$ is between 0 and 1 ).

- $1-\alpha$ is the effect rate of mobility direction changes in sampling time $t$ on the waking up cluster's size at sampling time $t+1$.

- $v_{\text {pre }}(t)\left|\tan \theta^{\prime}(t)\right|$ represents the deviation rate of target from the predicted path at sampling time $t$.

- $b$ and $c$ are constants.

Also figure 6 shows an approximation of waking up area in the sampling time $t+1$

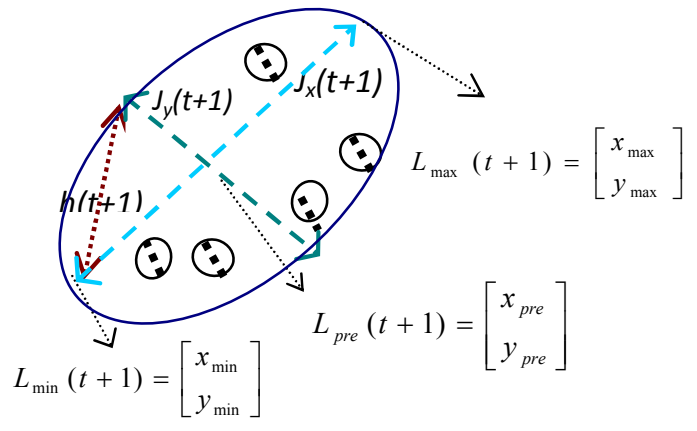

Fig. 6. Approximation of wakening up area in the sampling

$$
\text { time } t+1
$$

\subsection{The merging of backbone clusters}

After approximating the long and short diameters of wakening up area at sampling time $t+1$, by the center of the waked up cluster at sampling time t, $L_{\text {pre }}(t)$, this node sends a wake up message to the nearest $\mathrm{CH} 1$ node in $L_{\text {pre }}(t+1)$. This message includes some information such as $L_{\min }(t+1), L_{\max }(t+1)$ and $h(t+1)$ (this message has been shown in Figure 7). After the node placed at $L_{\text {pre }}(t+1)$ received this message, it subsequently broadcasts a wake up message to all of its peripheral backbone clusters. This message includes information such as $L_{\min }(t+1), L_{\max }(t+1), h(t+1)$ and ID (Figure 8).

By receiving this message each $\mathrm{CH} 1$ node first checks if its coordinates are inside the defined area or not, regarding $L_{\min }(t+1), L_{\max }(t+1)$ and $h(t+1)$. For example, suppose the coordinates of $\mathrm{i}$-th cluster head node, $\left(\mathrm{CH}_{i}\right)$ is $\left(x_{i}, y_{i}\right)$ and its distance to $L_{\min }(t+1)$ and $L_{\max }(t+1)$ is $d(\min , i)$ and $d(\max , i)$, respectively. So if its coordinates satisfy the equation11, which is defined based on mathematical concept of ellipse, then it wakes up all member nodes in its cluster and broadcasts the wake up message to its peripheral $\mathrm{CH} 1$ nodes.

$$
d(\min , i)+d(\max , i)<2 h(t+1)
$$

Where $h^{2}(t+1)$ is defined as

$$
h^{2}(t+1)=\left(\frac{J_{y}(t+1)}{2}\right)^{2}+\left(\frac{J_{x}(t+1)}{2}\right)^{2}
$$

The main point is that the goal of sending item ID (as one of the items of wake up message) to $\mathrm{CH} 1$ nodes is introduction of the second-level cluster head (CH2). Generally, in this method, if we consider the neighborhood degree, $q$, for each $\mathrm{CH} 1$ node, then the number of messages which are interchanged to introduce the $\mathrm{CH} 2$ node to all of $\mathrm{CH} 1$ nodes is $N=O(q k)$, where $k$ is the number of clusters which are going to be merged. Figure 9 shows $\mathrm{T}^{2} \mathrm{BM}^{2}$ method briefly.

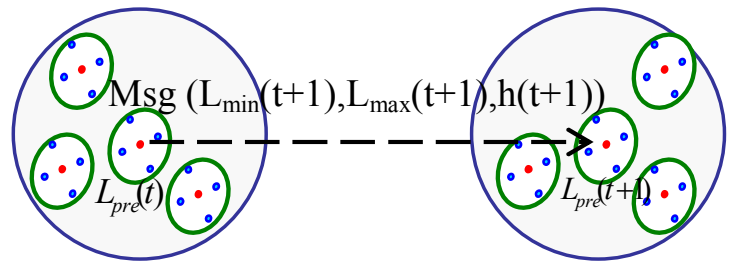
a) The waked up area in sampling time $t$
b) The wakening up area in sampling time $(t+1)$

Fig. 7. Sending the wake up message from the waked up area in sampling time $t$ to the center of the area that will be waked up in sampling time $(t+1)$

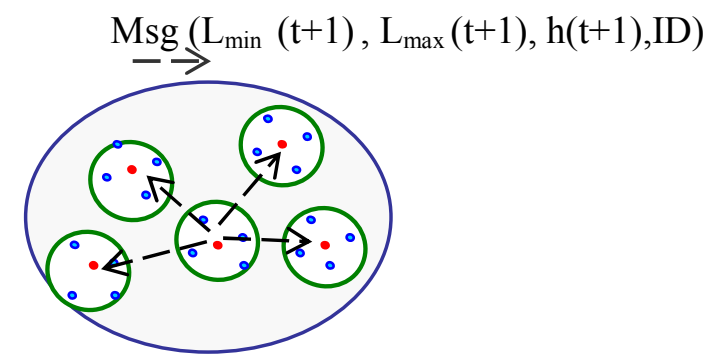

Fig. 8. Sending the wake up message from the center of area to all of peripheral $\mathrm{CH} 1$ nodes. 


\section{Stages of target tracking in $\mathrm{T}^{2} \mathrm{BM}^{2}$ method}

Step1: Estimating the target's location in sampling time $(t+1)$ (relation 6).

Step2: Calculating the long diameter of the area that would be waked up in sampling time $(t+1)$ regarding maximum and minimum target velocity (relation 9 ).

Step3: Calculating the short diameter of the area that would be waked up in sampling time $(t+1)$ regarding the short diameter of waked up area in sampling time $t$ and deviation rate of target from predicted path (relation 10).

Step4: Waking up the backbone clusters which are located in defined area (relation 11).

Step5: The waked up backbone clusters do sensing operations, and $\mathrm{CH} 1$ nodes transmit the integrated information of each backbone cluster to $\mathrm{CH} 2$.

Step6: After integrating the received information, $\mathrm{CH} 2$ node sends sampling results to the base station.

Fig.9. the stages of target tracking in $\mathrm{T}^{2} \mathrm{BM}^{2}$ method

\section{COMPARISON OF ENERGY CONSUMPTION IN SDC STRUCTURE WITH THE TRADITIONAL CLUSTERING STRUCTURES}

In this section the energy consumption in three structures, static clustering, dynamic clustering and SDC structure has been compared. We used a simple radio model to estimate the energy spent at the receiver and the transmitter. In every structure, it is assumed that $\mathrm{N}$ sensor nodes in average would participate in each step of tracking. In the following, we describe the energy consumption of each structure in detail.

\subsection{Static clustering method}

In this structure, we suppose that each static cluster has $\mathrm{N}$ sensor nodes. The consumed energy by a member node to transmit $l$ bit message to a cluster head is based on [13]:

$$
E_{N C H}=l\left(E_{\text {elec }}+E_{f s} d_{\text {toch }}^{2}\right) T_{\text {nchtx }}
$$

where $E_{\text {elec }}$ is the energy of electronic transmission/reception, $E_{f s}$ is the amplification factor, $d_{t o c h}$ is the distance between each member node and its cluster head, $T_{n c h t}$ is supposed to be the transmitting time for each member node to transmit $l$ bit message to a cluster head.

Also, the energy consumption by a cluster head is based on:

$E_{C H}=l E_{\text {elec }}(N-1) T_{\text {chrx }}+N l E_{d a}+\left(l E_{\text {elec }}+l E_{m p} d_{B S}^{4}\right) T_{\text {chtx }}$

where $l E_{\text {elec }}(N-1)$ is the energy consumed by a cluster head to receive $l$ bit message from $(N-1)$ member nodes and $T_{c h r}$ is the number of seconds for which the cluster head listens to one member node. $N l E_{d a}$ is the necessary energy for data aggregation and $\left(l E_{\text {elec }}+l E_{m p} d_{B S}^{4}\right) T_{c h t x}$ is the energy consumed for transmitting $l$ bit message to a base station. Where, $E_{m p}$ is the amplifier energy also $T_{\text {chtx }}$ is the number of seconds for which the cluster head transmits to base station. In static clustering, the total energy consumed in one round is the addition of $E_{N C H}$ and $E_{C H}$ as:

$$
E_{\text {STATIC }}=(N-1) E_{N C H}+E_{C H}
$$

\subsection{Dynamic clustering method}

In this structure, if we suppose each cluster which is formed dynamically is similar to static clustering and is composed of $N$ nodes, then the energy consumptions in member nodes and cluster heads are similar to static clustering. But this structure has extra energy consumption due to dynamic formation of clusters and cluster head election. If we suppose that in each stage, the cluster head node is elected using message interchanges, then the number of message interchanges is denoted by num_dynamic and estimated as:

$$
\text { num_dynamic }=N q
$$

where $q$ is the neighborhood degree of each member node. Supposing that messages which are interchanged between nodes for election of cluster head are similar to data messages, then the energy consumption for cluster head election is based on

$$
E_{C H-E L E C T}=n u m \_ \text {dynamic }\left(2 l E_{\text {elec }}+l E_{f s} d_{n c h-n c h}^{2}\right)
$$

where $d_{n c h-n c h}$ is the distance between two member nodes. In this structure, the total energy consumption in one round is estimated as equation (18), where, $E_{N C H}$ and $E_{C H}$ are estimated based on equations (13) and (14).

$$
E_{\text {DYNAMIC }}=E_{C H}+(N-1) E_{N C H}+E_{C H-E L E C T}
$$

\subsection{SDC method}

In this structure, we assume the backbone of the network has been created as static clustering, and each cluster is consisted of $M$ member nodes. Also similar to static and dynamic clustering, $N$ nodes are involved in each step of tracking. This structure has gained from this fact that, the number of message interchanges for cluster head election in new formed cluster is less than dynamic clustering structure. In other words, in dynamic clustering all member nodes compete to elect the cluster head node, but in this structure only first-level cluster head nodes do message interchanges. If we assume that the neighborhood degree of each $\mathrm{CH} 1$ node is $q$, then the number of message interchanges for introducing the second-level cluster head, Num_SDC, is given as:

$$
\text { Num_SDC }=k q
$$

where $k$ is the number of backbone clusters, which are going to be merged, and is given as: 


$$
k=\frac{N}{M}
$$

In this structure, the total energy consumption in each tracking step is the sum of energy consumption of member nodes in backbone clusters to transmit information to $\mathrm{CH} 1$ nodes, $E_{N C H}$, the energy consumption of $\mathrm{CH} 1$ nodes to transmit integrated information to $\mathrm{CH} 2$ node, $E_{C H 1}$, the energy consumption for message interchanges between $\mathrm{CH} 1$ nodes due to introduction of $\mathrm{CH} 2$ node, $E_{\mathrm{CH} 2 \text {-int ro }}$ and the consumed energy in the $\mathrm{CH} 2$ node, $E_{\mathrm{CH} 2}$.

In summary the total energy consumption in this structure is calculated as:

$$
E_{S D C}=(N-1) E_{N C H}+k E_{C H 1}+E_{C H 2 \text {-intro }}+E_{C H 2}
$$

where, similar to static clustering structure, $E_{N C H}$ is given as relation (13) and $E_{C H 1}$ is estimated by:

$$
\begin{aligned}
& E_{C H 1}=M l E_{\text {elec }} T_{\text {chrx }}+M l E_{D A}+ \\
& \left(l E_{\text {elec }}+l E_{f s} d_{C H 1-C H 2}^{2}\right) T_{C H 1-C H} 2
\end{aligned}
$$

where $M I E_{\text {elec }} T_{\text {CHRX }}$ is the consumed energy by each $\mathrm{CH} 1$ node to receive $l$ bit information from $M$ member nodes, $T_{c h r x}$ is the number of seconds for which each $\mathrm{CH} 1$ node listens to each member node and $M l E_{D A}$ is the energy used for data aggregation. $\left(l E_{\text {elec }}+l E_{f \mathrm{~s}} d_{\mathrm{CH} \mathrm{l}-\mathrm{CH} 2}^{2}\right) T_{\mathrm{CH} \mathrm{l}-\mathrm{CH} 2}$ is the consumed energy for transmitting the integrated information to $\mathrm{CH} 2$ node. Where $d_{C H 1-C H 2}$ is the distance between each $\mathrm{CH} 1$ node and $\mathrm{CH} 2$ node. $T_{C H 1-C H 2}$ is supposed to be the transmitting time for each $\mathrm{CH} 1$ node to transmit $l$ bits information to $\mathrm{CH} 2$ node. The expression for the energy consumption by a $\mathrm{CH} 2$ node is given as:

$$
\begin{aligned}
E_{C H 2}= & k l E_{\text {elec }} T_{t o C H 1}+k l E_{D A}+ \\
& \left(l E_{\text {elec }}+l E_{m p} d_{B S}^{4}\right) T_{C H 2-B S}
\end{aligned}
$$

where $k l E_{\text {elec }} T_{\text {toCH } 1}$ is the consumed energy to receive $l$ bits information from $\mathrm{CH} 1$ nodes, $T_{t o C H}$ is the number of seconds for which $\mathrm{CH} 2$ node listens to each $\mathrm{CH} 1$ node, and $k l E_{D A}$ is the energy used for data aggregation. Also $\left(l E_{\text {elec }}+l E_{m p} d_{B S}^{4}\right) T_{C H 2-B S}$ is the consumed energy to transmit integrated information to base station where $T_{C H 2-B S}$ is supposed to be the transmitting time for $\mathrm{CH} 2$ node to transmit $l$ bits information to it.

\section{PERFORMANCE EVALUATION OF THE PROPOSED METHOD}

We have used simulations to compare the accuracy of the tracking and the energy consumed by sensors. The velocity of target is modeled by the composition of Normal Distribution process and Gauss-Markov process as:

$v_{n}=\gamma\left(N\left(\mu_{v}, \sigma_{v}\right)\right)+(1-\gamma)\left(\alpha v_{n-1}+(1-\alpha) \mu_{v}\right)$

Where $v_{n}=v(n \Delta t)$ is the $\mathrm{n}$-th sample of target velocity, $\mu_{v}$ is the mean value of $v$, and $\sigma_{v}$ is the variance of $v$. The same equation holds for the target direction $\theta$, with corresponding values of $\theta_{n}, \mu_{\theta}$ and $\sigma_{\theta}$ as equation (25).

$\theta_{n}=\gamma\left(N\left(\mu_{\theta}, \sigma_{\theta}\right)\right)+(1-\gamma)\left(\alpha \theta_{n-1}+(1-\alpha) \mu_{\theta}\right)$

By coefficient $\gamma$, we can control target mobility model in simulations. In other words, if $\gamma=1$, the target mobility model is completely in Normal Distribution form (the target moves randomly). On the other side, $\gamma=0$ means the target is moving uniformly. Table1 illustrates the detailed configuration of our simulations for target mobility. The sensor network field is considered as a rectangular area of $200 \times 200 \mathrm{~m}^{2}$, where 1000 sensor nodes have been deployed in a grid structure. All nodes are identical and so they have the same initial resources. Table 2 illustrates the detailed configuration of simulations for energy consumption.

Table1. Simulation settings of target mobility

\begin{tabular}{|c|l|c|}
\hline Parameter & \multicolumn{1}{|c|}{ Description } & Setting \\
\hline$v_{1}$ & Initial velocity & $12 \mathrm{~m} / \mathrm{s}$ \\
\hline$\mu_{v}$ & Mean of velocity & $10 \mathrm{~m} / \mathrm{s}$ \\
\hline$\sigma_{v}$ & Standard deviation of velocity & $10 \mathrm{~m} / \mathrm{s}$ \\
\hline$\theta_{1}$ & $\begin{array}{l}\text { Angle of initial deviation from } \\
\text { anticipated path }\end{array}$ & 10 degrees \\
\hline$\mu_{\theta}$ & $\begin{array}{l}\text { Average of deviation angle from } \\
\text { anticipated path }\end{array}$ & 20 degrees \\
\hline$\sigma_{\theta}$ & $\begin{array}{l}\text { Standard deviation of deviation } \\
\text { angle }\end{array}$ & $\begin{array}{l}\text { The effect rate of size of wake up } \\
\text { cluster in sampling time } \mathrm{t} \text { on size } \\
\text { of wake up cluster in sampling } \\
\text { time } \mathrm{t}+1\end{array}$ \\
\hline$\alpha$
\end{tabular}


Table 2. Simulation settings of energy consumption

\begin{tabular}{|c|c|c|}
\hline Parameter & Description & Setting \\
\hline$E_{f s}$ & $\begin{array}{l}\text { The amplification factor for } \\
\text { near distances }\end{array}$ & $\begin{array}{c}10 \\
\text { nJoules/bit }\end{array}$ \\
\hline$E_{m p}$ & $\begin{array}{l}\text { The amplification factor for } \\
\text { far from distances }\end{array}$ & $\begin{array}{c}0.0013 \\
\text { pJoules/bit }\end{array}$ \\
\hline$E_{\text {elec }}$ & The electronic energy & 50 nJoules/bit \\
\hline$E_{d a}$ & $\begin{array}{l}\text { The necessary energy for } \\
\text { data aggregation }\end{array}$ & 5 nJoules/bit \\
\hline$T_{n c h t x}$ & $\begin{array}{l}\text { The transmitting time for } \\
\text { each member node to } \\
\text { transmit } l \text { bit message to } \\
\text { cluster head }\end{array}$ & $0.5 \mathrm{sec}$ \\
\hline$T_{\text {chrx }}$ & $\begin{array}{l}\text { The number of seconds for } \\
\text { which the cluster head } \\
\text { listens to one member node }\end{array}$ & $1 \mathrm{sec}$ \\
\hline$T_{\text {chtx }}$ & $\begin{array}{l}\text { The number of seconds for } \\
\text { which the cluster head } \\
\text { transmits to base station }\end{array}$ & $1 \mathrm{sec}$ \\
\hline$T_{\mathrm{CH} 1-\mathrm{CH} 2}$ & $\begin{array}{l}\text { The transmitting time for } \\
\text { each } \mathrm{CH} 1 \text { node to transmit } \\
l \text { bit information to } \mathrm{CH} 2 \\
\text { node }\end{array}$ & $0.5 \mathrm{sec}$ \\
\hline$T_{t o C H I}$ & $\begin{array}{l}\text { The number of seconds for } \\
\text { which } \mathrm{CH} 2 \text { node listens to } \\
\text { each } \mathrm{CH} 1 \text { node }\end{array}$ & $1 \mathrm{sec}$ \\
\hline$T_{C H 2-B S}$ & $\begin{array}{l}\text { The transmitting time for } \\
\mathrm{CH} 2 \text { node to transmit } l \text { bit } \\
\text { information to the base } \\
\text { station }\end{array}$ & $1 \mathrm{sec}$ \\
\hline$l$ & Message size & 6bit \\
\hline
\end{tabular}

We evaluated the proposed method by tracking error and energy consumption with an inadaptable target tracking protocol based on static clustering structure [6], and the target tracking protocol based on dynamic clustering structure in [5]. To implement different mobility models, we increase the coefficient $\gamma$ continuously in relations (24) and (25). In fact, we increase target random mobility by the increment of $\gamma$. In diagrams of simulation results, $\mathrm{SC}$ is the inadaptable target tracking protocol based on static clustering in [5], DC is the target tracking protocol based on dynamic clustering in [6], and SDC is based on $\mathrm{T}^{2} \mathrm{BM}^{2}$ method which uses the semi-dynamic clustering structure.

As we see in Figure 10, when the target moves with a low random mobility coefficient, tracking errors in the three methods are approximately similar. Recalling that, in SDC method in order to decrease the energy consumption, the number of nodes participating in tracking will be decreased. This causes a little increment in the tracking error. However, when $\gamma$ increases, the tracking error in SDC method will be less than static and dynamic clustering structures due to the increment of nodes participate in tracking and the better selection of nodes for being waked up based on the target mobility model. On the other hand, when the coefficient $\gamma$ increases, the energy consumption of SC structure is approximately constant. But in dynamic clustering structure the energy consumption will raise gradually due to the reclustering needs. Also when the random mobility coefficient is low $(\gamma<0.3)$, the SDC structure has lower energy consumption because of decreasing the size of clusters. However, by the increment of $\gamma$, more nodes participate in tracking and these results to the increment of energy consumption. The important point is that up to random mobility coefficient 0.7 , energy consumption of SDC is less than dynamic clustering structure because of the lower message interchanges needed to select cluster head. But after that, the energy consumption will be more than dynamic clustering structure due to the increment of clusters size (Figure 11). Also, Figures 12 and 13 show the changes of tracking errors and energy consumptions of the three clustering structures with $\gamma=0.2$ gradually by passing the time. As we see in Figure 13, energy consumption of SDC will decrease rapidly because of the decrement of clusters size. This causes a little increment in the tracking error (Figure 12). Similarly, Figure14 shows the various speeds. As we see in Figure14, the increment of the tracking error in SDC happens slower than the other structures.

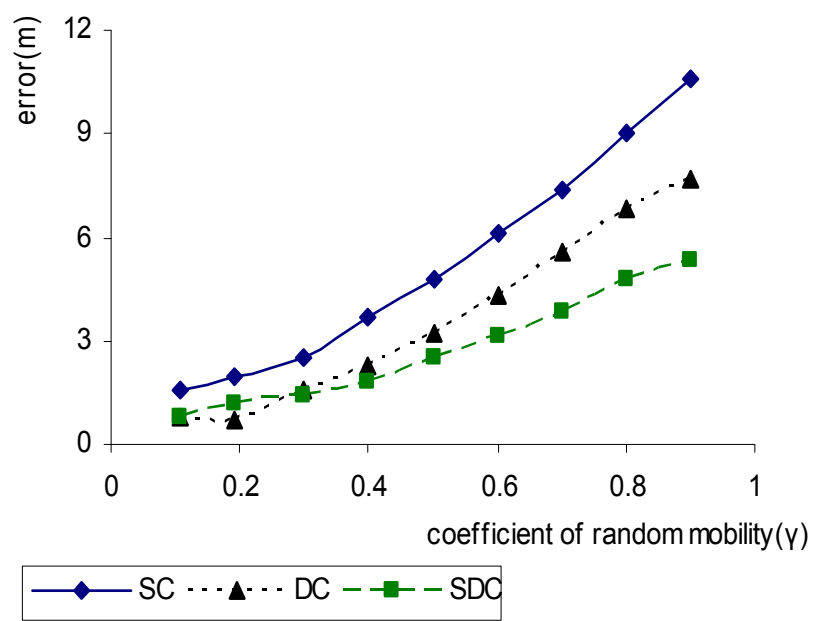

Fig. 10. Tracking error in different mobility models. 


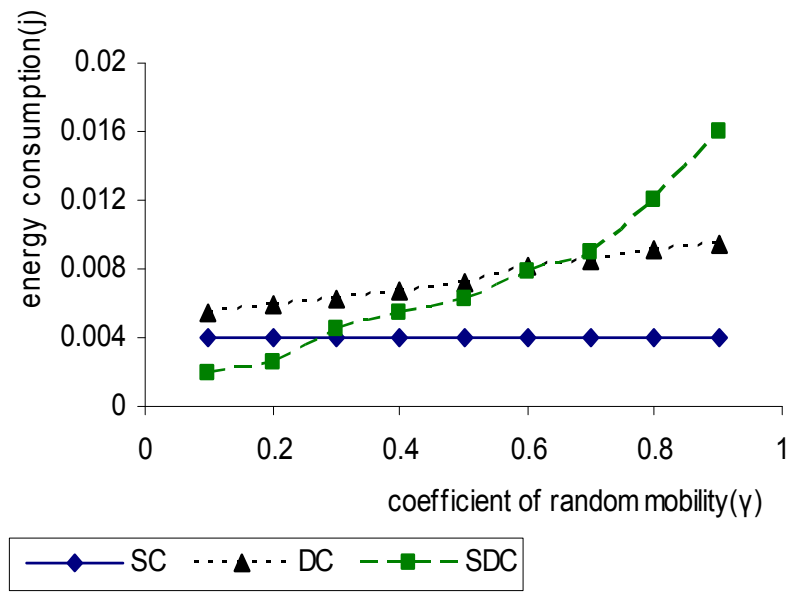

Fig. 11. Energy consumption in different mobility models

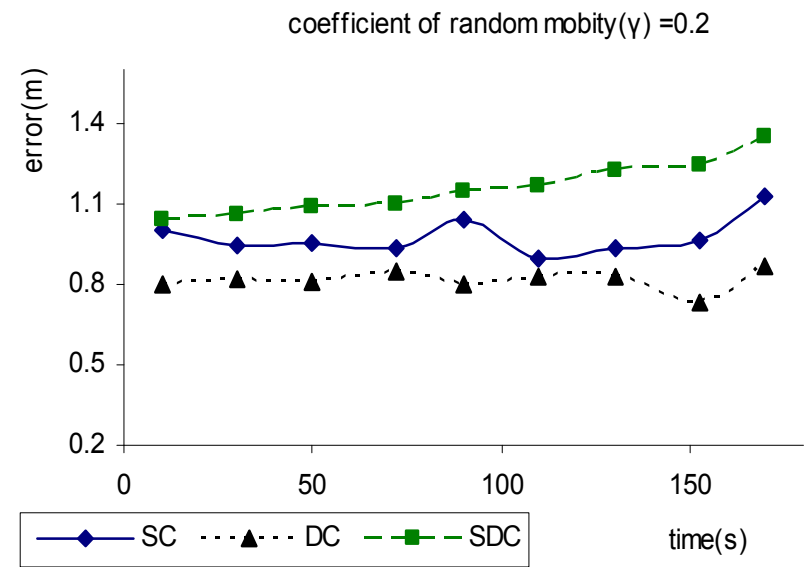

Fig. 12. Tracking error in different clustering structures with $\gamma=0.2$

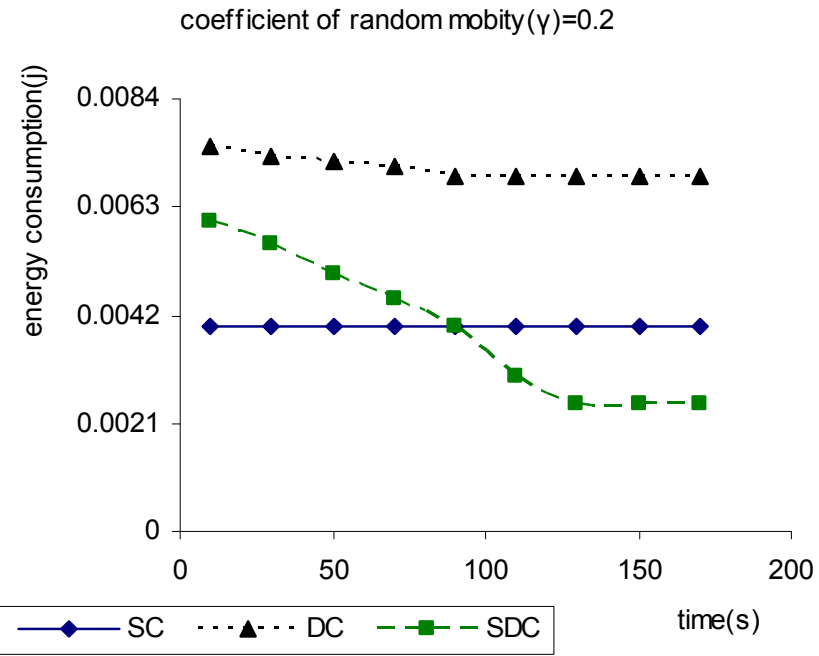

Fig. 13. Energy consumption when the target moves with $\gamma=0.2$

\section{CONCLUSION}

One of the important applications of wireless sensor network is tracking mobile targets. In this paper, we proposed a new target tracking method which is adaptive with target mobility model. In this method, we used two tools to adapt the size and shape of clusters with target mobility model. We implemented these tools by using a semi dynamic clustering structure which is adaptive with target mobility model. In this method, the shape of clusters is considered in elliptical form, which the short diameter

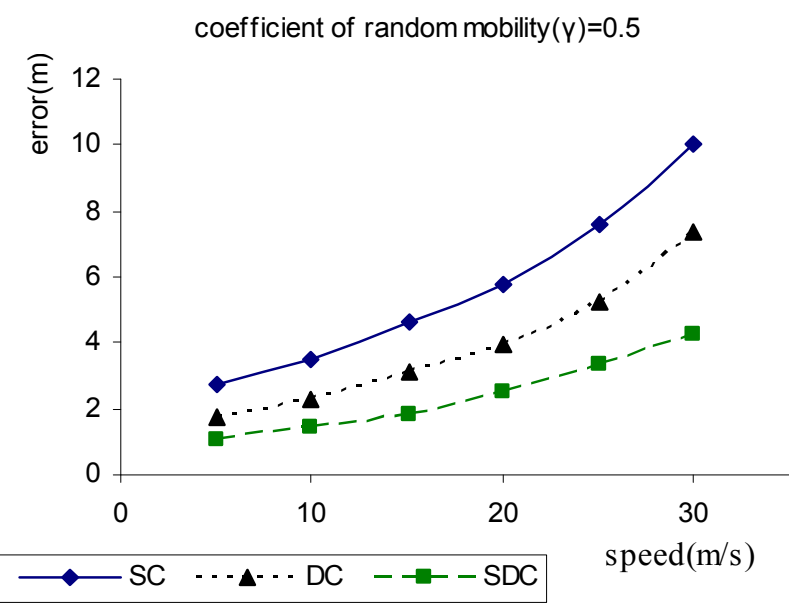

Fig. 14. Tracking error in different structures when the target moves with various speeds

depends on the deviation rate of target from predicted path and the long diameter depends on maximum and minimum velocity of target. In other words, in this method the size of waking up area depends on the coefficient of random mobility of target. It is proposed toward another possible area of work to estimate the size and shape of clusters more accurately based on more technical analysis of target mobility. 


\section{REFERENCES}

[1] H. W. Tsai, C. P. Chu, T. S. Chen, "Mobile object tracking in wireless sensor networks," Computer Communication, 2007, 30(8): 1811-1825.

[2] R. Gupta; S. R. Das. "Tracking Moving Targets in a Smart Sensor Network," IEEE Veh.Technol. Conf. 2003; pp. 3035-3039.

[3] W. Zhang; G. Cao. "DCTC: Dynamic convoy tree-based collaboration for target tracking in sensor networks," IEEE Transactions on Wireless Communications ,vol. 3, Sept. 2004.

[4] W.-P. Chen; J. C. Hou; and L. Sha. Dynamic clustering for acoustic target tracking in wireless sensor networks," in Proc. 11th IEEE International Conference on Network Protocols 2003, pp. 284-294.

[5] Guang-yao Jin, Xiao-yi Lu and Myong-Soon Park, "Dynamic Clustering for Object Tracking in Wireless Sensor Networks," 2006 International Symposium on Ubiquitous Computing Systems (UCS 2006), pp.200-209, Seoul, Korea, Oct. 11 13, 2006, (LNCS)(SCIE).

[6] Rahman, O., Choi, B.G., Monowar, M., Hong, C.S., “A Density Based Clustering for Node Management in Wireless Sensor Network," Springer Verlag LNCS 2007, $4773,527-530$.

[7] Vincent S. Tseng; Eric Hsueh-Chan Lu. "Energy-efficient real-time object tracking in multi-level sensor networks by mining and redicting movement patterns," The Journal of Systems and Software 2008.

[8] H, Yang; B. Sikdar."A protocol for tracking mobile targets using sensor networks." Proc. IEEE International Workshop on Sensor Networks Protocols and Applications, Anchorage, AK, 2003, pp. 71-81.

[9] In-Sook Lee; Zhen Fu; WenCheng Yang; Myong-Soon Park. "An Efficient Dynamic Clustering Algorithm for Object Tracking in Wireless Sensor Networks," The 2nd International Conference on Complex Systems and Applications, June. 8-10,2007, pp.1484-1488.

[10] Sara Pino-Povedano, Francisco-Javier Gonzalez Serrano, "Distributed Tracking and Classification of Targets with Sensor Networks," IEEE 2009.

[11] Guang-yao, J.; Xiao-yi, L.; Myong-soon, P. "Dynamic Clustering for object. tracking in wireless sensor networks," Proceeding of 3rd International Symposium on Ubiquitous computing systems, Seoul, , 2006; pp. 200-209.

[12] HyunSook, K; Eunhwa, K; Kijun,H. An Energy Efficient Tracking Method in Wireless Sensor Networks. In Next Generation Teletraffic and Wired/Wireless Advanced Networking, Publisher: Springer Berlin / Heidelberg, 2006;4003, pp. 278- 286

[13] Limin Meng; Kai Zhou; Jingyu Hua, Zhijiang Xu.” A Dynamic Clustering-Based Algorithm for Wireless Sensor Networks," International Symposium on Computer Science and Computational Technology2008; pp. 720-723. 